\title{
Intracranial intraaxial cerebral tufted angioma: case report
}

\author{
*Randy S. D'Amico, MD, ${ }^{1}$ George Zanazzi, MD, PhD, ${ }^{2}$ Gunnar Hargus, MD, PhD, ${ }^{2}$ \\ Timothy Dyster, BS, ${ }^{1}$ Shirley Chan, MD, ${ }^{3}$ Angela Lignelli-Dipple, MD, ${ }^{3}$ Tony J. C. Wang, MD, ${ }^{4}$ \\ Phyllis L. Faust, MD, PhD, ${ }^{2}$ and Guy M. McKhann II, MD'
}

Departments of ${ }^{1}$ Neurological Surgery, ${ }^{2}$ Pathology and Cell Biology, ${ }^{3}$ Radiology, and ${ }^{4}$ Radiation Oncology, Columbia University Medical Center, New York, New York

\begin{abstract}
Tufted angioma (TA) is a rare, slow-growing, vascular lesion that commonly presents as a solitary macule, papule, or nodule arising in the soft tissues of the torso, extremities, and head and neck in children and young adults. Adult-onset cases have been infrequently reported. While typically benign, TAs may be locally aggressive. Complete physical examination and hematological workup are recommended in patients with TA to exclude the presence of Kasabach-Merritt phenomenon (KMP). The authors describe the case of a 69 -year-old man with a contrast-enhancing frontal lobe lesion, with surrounding vasogenic edema, which was treated by gross-total resection. Characteristic histological features of a TA were demonstrated, with multiple cannonball-like tufts of densely packed capillaries emanating from intraparenchymal vessels in cerebral cortex and adjacent white matter. Tumor recurrence was detected after 4 months and treated with adjuvant Gamma Knife radiosurgery. To the extent of the authors' knowledge, this case illustrates the first report of TA presenting in an adult as an intracranial intraaxial tumor without associated KMP. The fairly rapid regrowth of this tumor, requiring adjuvant treatment after resection, is consistent with a potential for locally aggressive growth in a TA occurring in the brain.
\end{abstract}

https://thejns.org/doi/abs/10.3171/2016.10.JNS162207

KEY WORDS tufted angioma; brain tumor; intracranial; Kasabach-Merritt phenomenon; kaposiform hemangioendothelioma; vascular disorders

$\mathrm{T}$ UfTED angioma (TA) is a rare, slow-growing vascular tumor derived from capillary and lymphatic endothelium. Originally, TA was termed an angioblastoma ${ }^{17}$ or progressive capillary hemangioma. ${ }^{14}$ In 1976 , Jones coined the name "tufted angioma" based on its histological features of multiple, discrete, cannonball-like lobules ("tufts") of densely packed capillaries surrounded by crescentic spaces. ${ }^{6}, 12$ TA usually presents as a solitary, expanding, erythematous, or violaceous soft-tissue macule, papule, or plaque. ${ }^{19}$ Generally, TA is recognized as a benign lesion that may nonetheless be locally aggressive but without reported evidence of distant metastases. However, multifocal cases have been reported. ${ }^{20}$ Children and young adults are predominantly affected by TA, with adult onset described infrequently., ${ }^{9}, 20$

To date, the etiology and pathogenesis of TA remain uncertain, although some believe TA and kaposiform hemangioendothelioma (KHE) are part of a continuum of the same pathological process. ${ }^{9,19}$ Interestingly, both TA and KHE may be associated with Kasabach-Merritt phenomenon (KMP), a clinical constellation of thrombocytopenia, consumptive coagulopathy, and purpura associated with larger lesions $(>5 \mathrm{~cm}) .8,9,19,21$

Together, TA/KHE have been known to arise in a number of anatomical locations including the soft tissues of the extremities, the torso, mediastinum, retroperitoneum, head and neck region, and rarely oral mucosa. ${ }^{5,9}$ Cases of KHE involving the internal/external auditory canals and the tentorium cerebelli, without evidence of parenchymal involvement, have been reported. ${ }^{2,3,10}$ To our knowledge, no reports exist of TA/KHE arising within brain parenchyma. In this paper we describe the case of a 69 -year-

ABBREVIATIONS GFAP = glial fibrillary acidic protein; GKRS = Gamma Knife radiosurgery; KHE = kaposiform hemangioendothelioma; KMP $=$ Kasabach-Merritt phenomenon; $T A=$ tufted angioma.

SUBMITTED August 24, 2016. ACCEPTED October 21, 2016.

INCLUDE WHEN CITING Published online February 24, 2017; DOI: 10.3171/2016.10.JNS162207.

* Drs. D'Amico and Zanazzi contributed equally to this work; and Drs. Faust and McKhann contributed equally to this work. 

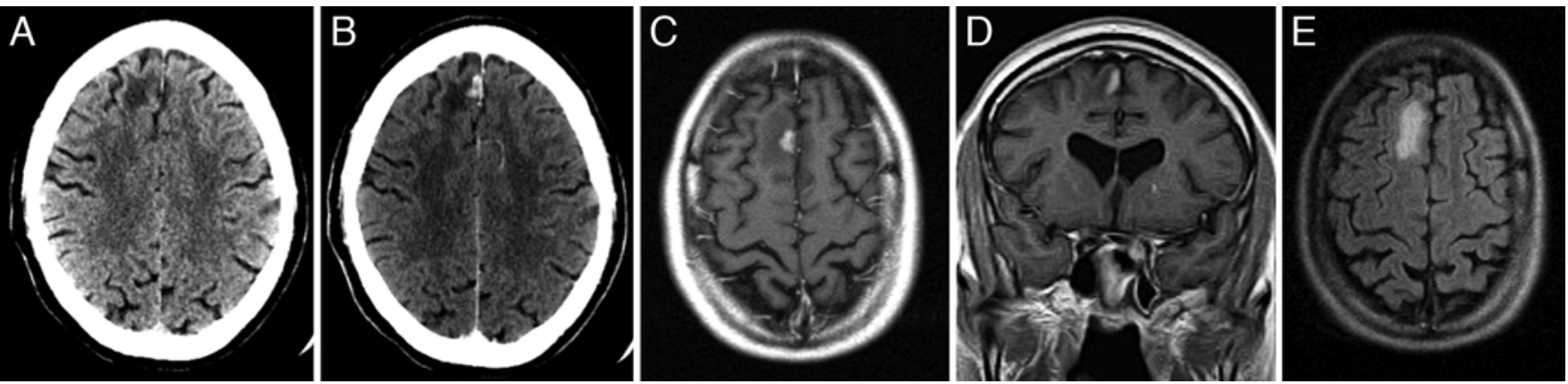

FIG. 1. Imaging features of intracranial TA. CT (A and B) and MR (C-E) images with and without contrast were obtained 15 and 11 weeks, respectively, prior to surgery. Precontrast (A) and postcontrast $(B)$ head CT scans demonstrate a hypodense lesion in the medial right superior frontal lobe with avid curvilinear enhancement. Postcontrast axial (C) and coronal (D) MR images demonstrate an intraaxial, curvilinear, enhancing right parasagittal frontal lobe mass involving the cortex and subcortical white matter with surrounding associated hyperintense T2 FLAIR signal (E) suggestive of associated edema.

old man with a medial frontal lobe intraaxial TA without associated KMP, treated using gross-total resection and subsequent adjuvant Gamma Knife radiosurgery (GKRS).

\section{Case Report}

History and Examination

A 69-year-old man presented with mild subjective memory complaints. There was no family history of hereditary disease or malignancy. No laboratory abnormality was found, including any platelet abnormality. Precontrast CT of the head demonstrated a hypodense lesion in the right superior medial frontal lobe with no evidence of hemorrhage or calcification (Fig. 1A). Postcontrast CT demonstrated avid curvilinear enhancement located medially (Fig. 1B). There was no bone involvement. Brain MRI was notable for a right frontal, parasagittal, avidly enhancing lesion lying flat along the falx cerebri and involving the cortex and subcortical white matter (Fig. 1C and D). The enhancement pattern was noted to be curvilinear and ribbon-like, outlining the cortex. A hyperintense T2 FLAIR signal was noted surrounding the enhancing lesion and confined to the right superior frontal lobe with mild associated gyral thickening, suggesting the presence of surrounding vasogenic edema (Fig. 1E). There was no associated diffusion restriction or susceptibility. The initial differential diagnosis included: dural-based lesions such as meningioma, given its proximity to the falx; brain metastasis, although the patient had no evidence of systemic cancer; cortically based enhancing lesions such as encephalitis, inflammation, subacute infarct, and seizures; and low-grade neoplasms such as dysembryoplastic neuroepithelial tumor, ganglioglioma, pilocytic astrocytoma, and pleomorphic xanthoastrocytoma.

\section{Operation}

Repeat imaging after 3 months revealed that while the amount of edema was similar in comparison with the prior scan, the lesion had enlarged by $2.5 \mathrm{~mm}$ in the axial plane and $1.0 \mathrm{~mm}$ in the coronal plane (Fig. 2). Surgery was performed using a right parasagittal craniotomy with frameless stereotactic guidance. Dissection was carried down along the interhemispheric surface of the right medial frontal lobe until obvious pathological tissue was visually identified. Of note, there was no dural attachment of the tumor. Intraoperative ultrasound was used to confirm the location of the lesion, combined with frameless stereotactic navigation. Subsequently, the lesion was entered and debulked. An intraoperative frozen-section diagnosis of angiocentric neoplasm was rendered (data not shown). The mass was circumferentially dissected and removed except for a small portion anteriorly attached to a prominent artery and vein sulcal complex. These vessels were
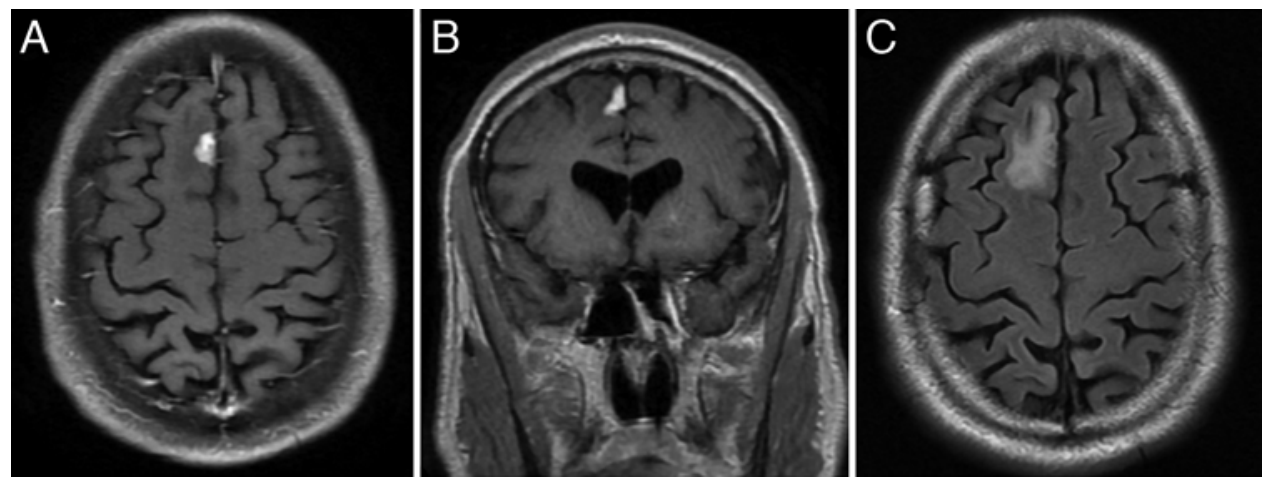

FIG. 2. Serial imaging demonstrates progression of intracranial TA. Postcontrast axial (A) and coronal (B) MR images obtained 4 weeks prior to surgery demonstrate interval progression of enhancement with stable surrounding edema, as represented by hyperintense T2 FLAIR signal (C). 


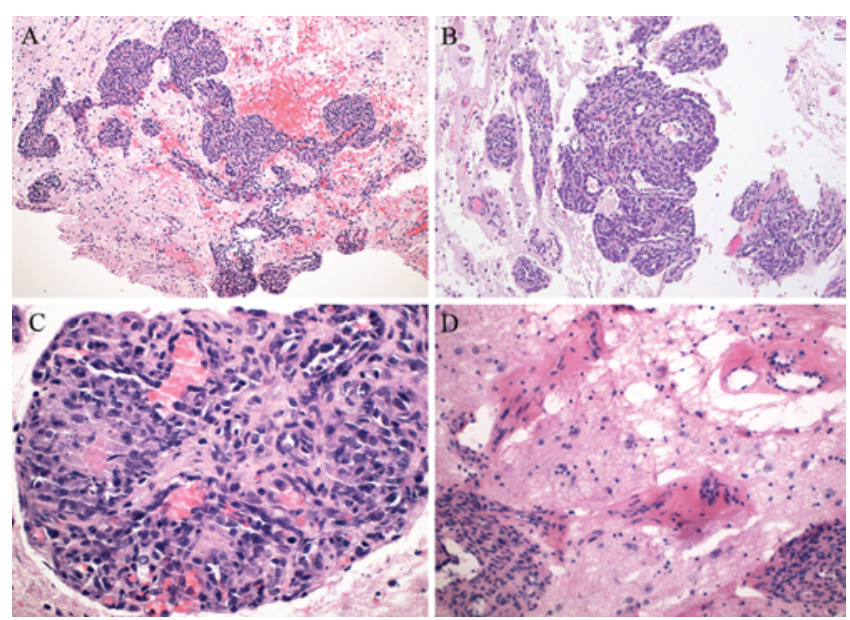

FIG. 3. Histological features of an intracranial TA. Low-magnification $(\times 100$; $A$ and $B)$ and high-magnification $(\times 400 ; C$ and D) photomicrographs of $\mathrm{H} \& \mathrm{E}$-stained sections show a vascular lesion with a nested architecture emanating from parenchymal vessel walls (A). The nests contain spindle-shaped endothelial cells associated with slit-like vascular channels (B). Intervening cells have oval and elongated nuclei with fine chromatin; apoptotic cells and rare small foci of necrosis are noted (C). In some areas, vessel walls are sclerotic (D). Figure is available in color online only.

preserved, and microdissection techniques were applied until there was no identifiable tumor remaining. However, residual microscopic disease attached to these vessels was likely.

\section{Histological Analysis}

Sections of the lesion stained with $\mathrm{H} \& \mathrm{E}$ showed a vascular neoplasm of moderate cellularity growing in small, tightly packed, rounded nests ("tufts") of angiomatous proliferations along parenchymal vessels in cerebral cortex and the adjacent white matter (Fig. 3A and B). Each nest contained clusters of plump endothelial cells surrounding small, slit-like capillary lumina (Fig. 3C). Cells surrounding the vascular channels had oval- to spindle-shaped nuclei with fine chromatin and only mild nuclear pleomorphism, and lacked epithelioid features. Apoptotic cells and rare small foci of necrosis were noted (Fig. 3C). In some foci, vessel walls were partially or completely sclerotic (Fig. 3D). The endothelial cells showed immunoreactivity for several markers, including CD31, CD34, FLI1 (Fig. 4A-C), and focally for GLUT1 (likely representing feeding vessels, data not shown). Cells in the nests were otherwise faintly positive for progesterone receptor, and negative for STAT6, inhibin A, pan-cytokeratin, epithelial membrane antigen, and D2-40 (data not shown). Many of the cells interspersed between vascular channels appeared to be pericytes and labeled with anti-smooth muscle actin (Fig. 4D). Scattered mitotic figures were noted, and Ki 67 labeling ranged from $9.5 \%$ to $25.6 \%$ of cells (Fig. 4E). In addition, a mixed inflammatory infiltrate was noted, including CD68positive histiocytes and CD45-positive lymphocytes observed between neoplastic cells within the nests (data not shown). The adjacent brain parenchyma showed marked reactive gliosis, including astrocytes highlighted by an immunostain for glial fibrillary acidic protein (GFAP; Fig. 4F) and CD68-positive microglia (data not shown).

\section{Postoperative Course}

Postcraniotomy, the patient was maintained on a 1-week steroid taper. Postresection MRI demonstrated gross-total resection with no residual contrast enhancement in the surgical bed (Fig. 5) and mildly reduced T2 FLAIR signal within the surrounding parenchyma. As the potential biological behavior of an intracranial intraaxial TA is unclear, routine surveillance MRI in 3-4 months was recom-

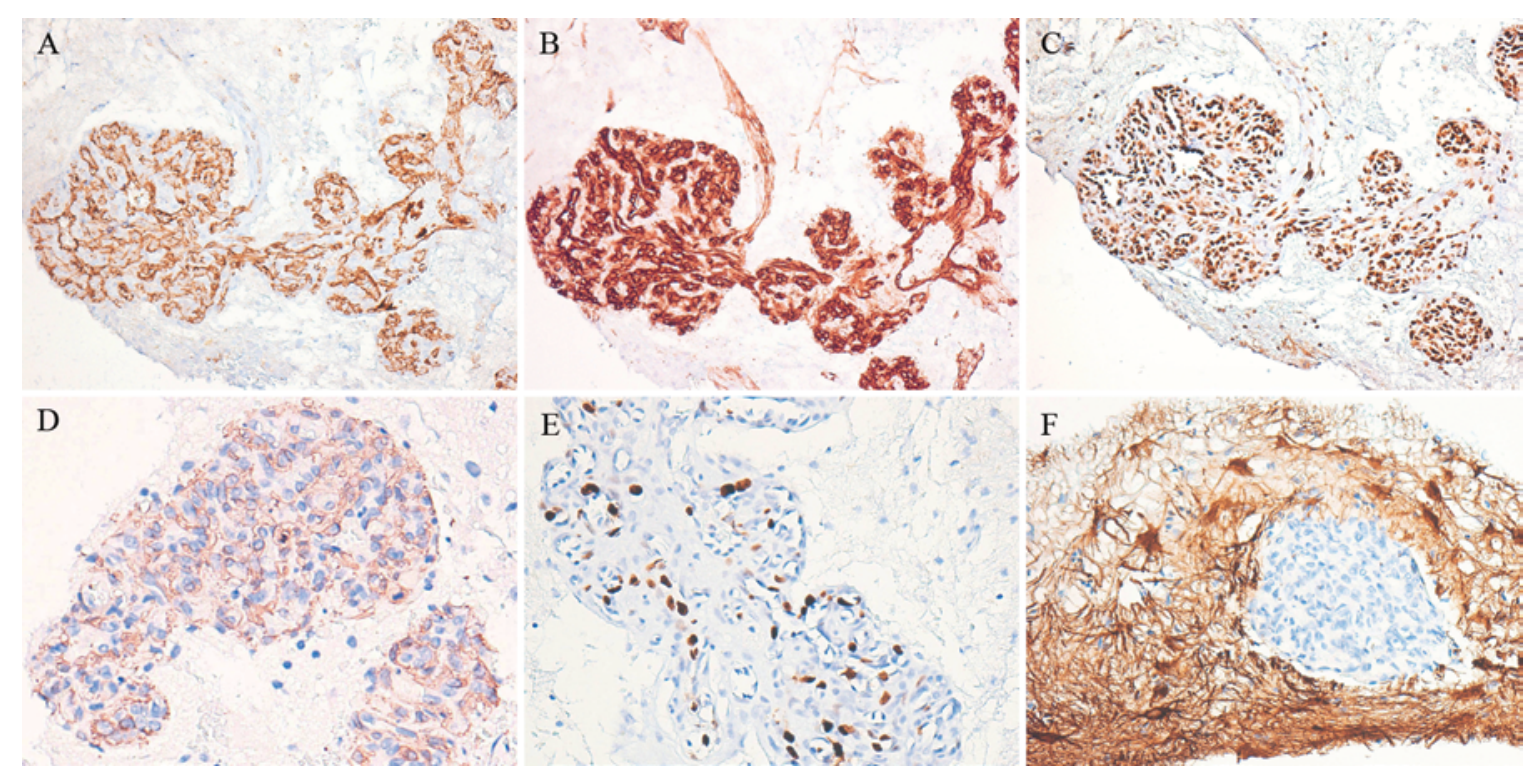

FIG. 4. Immunohistochemical characterization of an intracranial TA. Sections stained with anti-CD31 (A), anti-CD34 (B), and anti-FLI1 (C) reveal plasma membrane, cytoplasmic (A and B), and nuclear immunoreactivities (C) of these markers in endothelial cells, respectively. Anti-smooth muscle actin highlights pericytes in the nests (D). Ki 67 labeling is high in the lesion (E). The brain parenchyma surrounding the nests shows marked gliosis, which is highlighted by a GFAP immunostain $(F)$. Original magnification $\times 100(A-C), \times 400(D-F)$. Figure is available in color online only. 

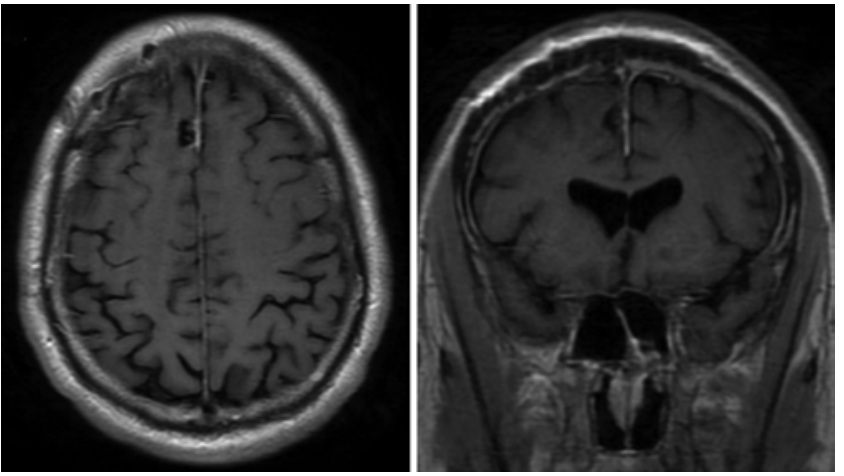

FIG. 5. Postresection postcontrast axial (left) and coronal (right) MR images demonstrate no residual contrast enhancement in the surgical bed.

mended to monitor for recurrence or progression. The patient initially did well postoperatively with no neurological sequelae. However, follow-up MRI performed 4 months after resection demonstrated clear recurrence along the posterior and inferior edge of the resection bed extending along the coronal plane inferior to the surgical bed (Fig. 6). The decision was made to pursue GKRS for the recurrent disease. A single treatment plan (Fig. 7) was devised to encompass the area of recurrence along the coronal plane inferior to the margin of the old resection bed, as well as incorporating the entirety of the old surgical bed and dural margin to minimize the risk of tumor progression. Target volume was $607.8 \mathrm{~mm}^{3}$. Given the irregular shape of the lesion tracking along the interhemispheric fissure and abutting the resection cavity, a total of 12 4-mm shots with sector blocking were outlined to deliver 18 Gy to the $50 \%$ isodose line. Dosimetry was determined in keeping with that of a more aggressive metastatic tumor rather than a more benign lesion, given the relatively rapid tumor progression and high Ki 67 index. Treatment was tolerated well. Repeat MRI performed 4 months after GKRS demonstrated no interval changes in the T2 FLAIR signal abnormality surrounding the lesion or in the enhancing lesion adjacent to the resection cavity, suggesting stable disease (Fig. 8). Again, a follow-up plan was made to repeat surveillance MRI in an additional 3-4 months to monitor for regression, recurrence, or progression. At last followup, occurring 8 months after initial resection, the patient remained at his neurological baseline with no clinical or radiographic signs of progression.

\section{Discussion}

In this paper we describe, to the best of our knowledge, the first case of an intracranial intraaxial TA. The differential diagnosis for TA includes KHE, capillary hemangioma, infantile hemangioma, glomeruloid hemangioma, vascular malformations, Kaposi sarcoma, hemangioblastoma, and hemangiopericytoma. Rare reports do exist of KHE arising from the temporal bone and the tentorium cerebel$\mathrm{li}^{2,3,10}$ and these typically show locally invasive growth. TA is generally regarded as closely related to KHE, named for its histological resemblance to Kaposi sarcoma and also containing infiltrating nodules with slit-like or crescentic vessels lined by spindled endothelial cells. ${ }^{5,7}$ Some believe that TA and KHE are part of a continuum of the
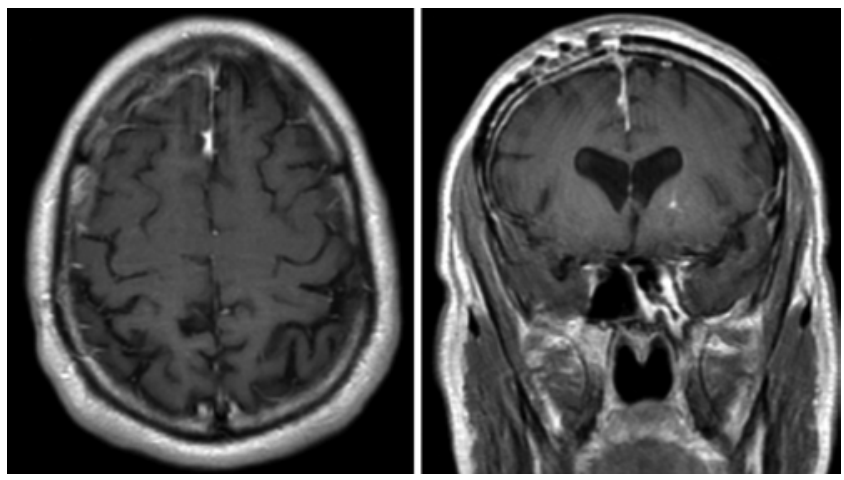

FIG. 6. Imaging features of recurrent TA. Postcontrast axial (left) and coronal (right) MR images demonstrate a recurrent enhancing lesion at the posterior inferior tumor bed with extension in the coronal plane.

same pathological process. The lesions of KHE appear very similar histologically to those of TA, but are more infiltrative with larger coalescing nodules. In addition, the lesions of KHE also contain epithelioid cells. ${ }^{4}$ In both lesions, platelet-rich fibrin thrombi may accumulate, leading to KMP. However, KMP develops in more than $70 \%$ of patients with $\mathrm{KHE}^{5}$ and in only $10 \%$ of patients with TA. ${ }^{13}$ In the present case, there were no associated laboratory abnormalities concerning for KMP.

Capillary hemangiomas are also common cutaneous lesions, but they have only rarely been reported in the brain, where they predominantly occur in an extraaxial location with circumscribed growths of capillaries surrounded by fibrous septae. ${ }^{1}$ In a systematic review of the literature, Mirza et al. found only 3 intracerebral cases of capillary hemangiomas among 14 adult patients. ${ }^{16}$ However, none of these intraaxial capillary hemangiomas exhibited a lobular architecture; rather, they had a papillary pattern and areas with capillaries dispersed in an edematous collagenous stroma. ${ }^{1}$ Other variants of hemangiomas in the differential diagnosis include infantile hemangiomas and glomeruloid hemangiomas. Infantile hemangiomas consist of closely packed capillary spaces with plump endothelial cells that express GLUT1; ${ }^{18}$ however, they do not display a cannonball-like architecture as in the present case. Glomeruloid hemangiomas, which are aggregates of vessels that resemble renal glomeruli, are usually associated with POEMS syndrome (polyneuropathy, organomegaly, endocrinopathy, monoclonal gammopathy, and skin changes) and Castleman disease. Kaposi sarcoma lesions show jagged, infiltrative, interconnected vascular channels with interspersed plasma cells. ${ }^{11}$ A diagnosis of hemangioblastoma or hemangiopericytoma was excluded by negative immunostains for inhibin A and STAT6, respectively. Taken together, the morphological and immunophenotypic features of this lesion are most consistent with TA.

The lesion in the present case had mitotic figures and an elevated Ki 67 index, indicative of a proliferative process. This supports the evidence of tumor progression witnessed on serial MRI. Of note, an elevated proliferation index is not unusual in $\mathrm{TA}^{15}$ or KHE, ${ }^{3}$ and does not by itself lead to a diagnosis of angiosarcoma. In the current case, the tumor's discrete growth pattern and lack of marked cellular atypia do not support a diagnosis of angiosarcoma. While 


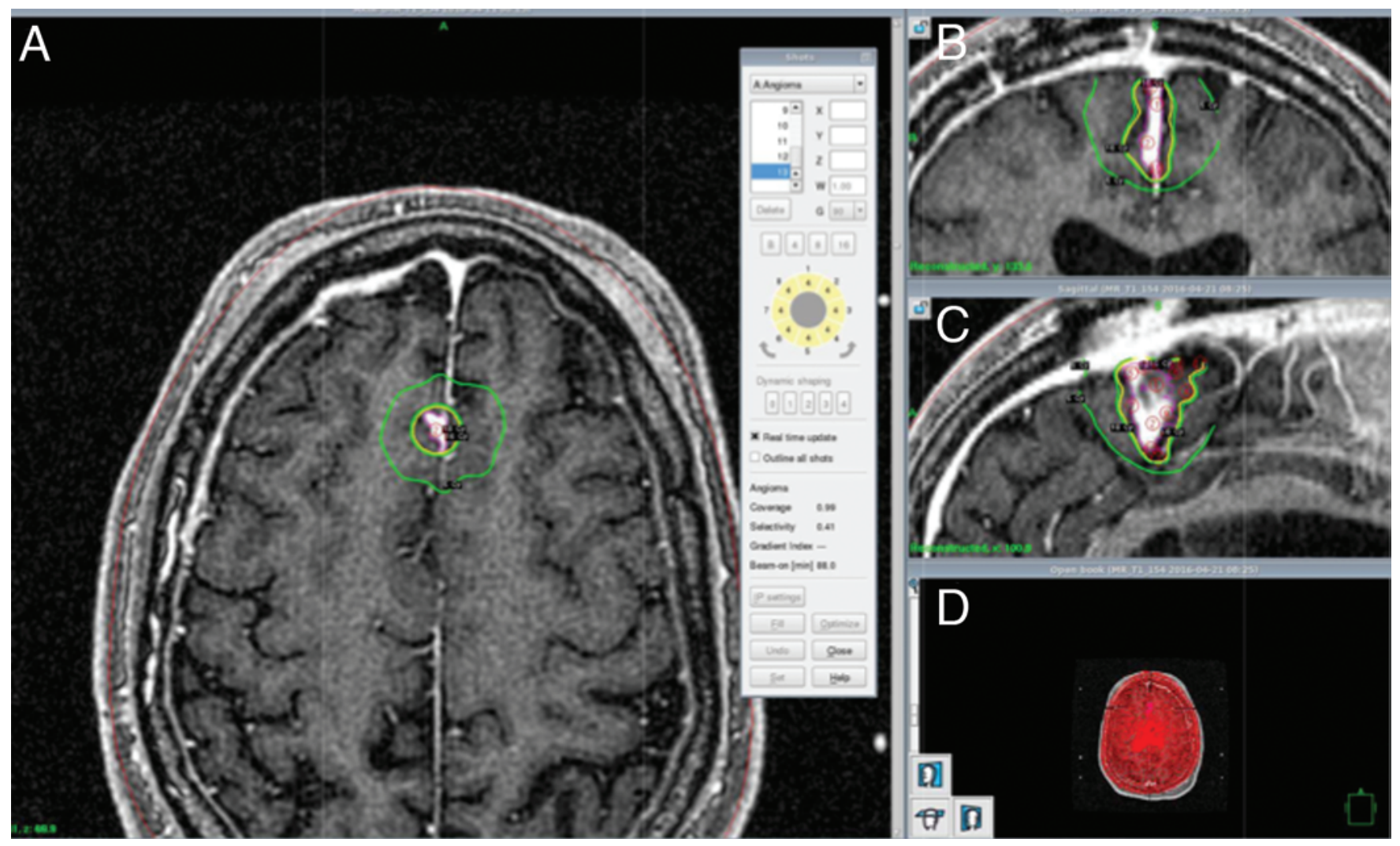

FIG. 7. GKRS plan. A single treatment plan was devised to encompass the area of recurrence along the axial (A), coronal (B), and sagittal (C) planes inferior to the margin of the old resection bed, as well as incorporating the entirety of the old surgical bed and dural margin to minimize the risk of tumor progression. Target volume was $607.8 \mathrm{~mm}^{3}$. Given the irregular shape of the lesion tracking along the interhemispheric fissure and abutting the resection cavity, a total of $124-\mathrm{mm}$ shots with sector blocking were outlined to deliver $18 \mathrm{~Gy}$ to the $50 \%$ isodose line. A 3D rendering of the skull is included in the plan, with red representing the assumed shape of the skull for dosimetry purposes (D). The rectangle in panel A is the "shot view" and shows the 13th shot is not set, i.e., there are only 12 shots set. Figure is available in color online only.

microscopic foci of residual TA after surgery may have allowed for recurrence in the absence of adjuvant therapy, the fairly rapid regrowth of this lesion within 4 months is consistent with the known locally aggressive growth potential for TA.

TA is a rarely described entity, and treatment modalities have been determined without randomized controlled trials or prospective studies. Single case reports and several small series form the foundation for recommendations, which include observation, surgery, medical therapies, arterial embolization, pneumatic compression, and radiation. Complete resection, however, remains the treatment of choice for small, localized lesions. ${ }^{19}$ Postoperative surveillance is recommended as recurrence of both TA and KHE has been reported. ${ }^{3,6}$ Malignant transformation of these lesions has never been reported. No evidence currently exists regarding the efficacy of GKRS for these lesions.

\section{Conclusions}

We have presented a case of TA, with no associated laboratory abnormalities concerning for KMP, arising within the parenchyma of the right frontal lobe. The tumor rapidly recurred after radiographically confirmed complete resection requiring adjuvant GKRS. This is the first reported case of this type of tumor in an intracranial, intraaxial location. In addition, this report provides a novel description of the morphological growth pattern and immunophenotypic features of an unusual intraaxial angioma. TA should be considered in the differential diagnosis of intracranial, intraaxial vascular tumors and resection should be considered the treatment of choice, particularly
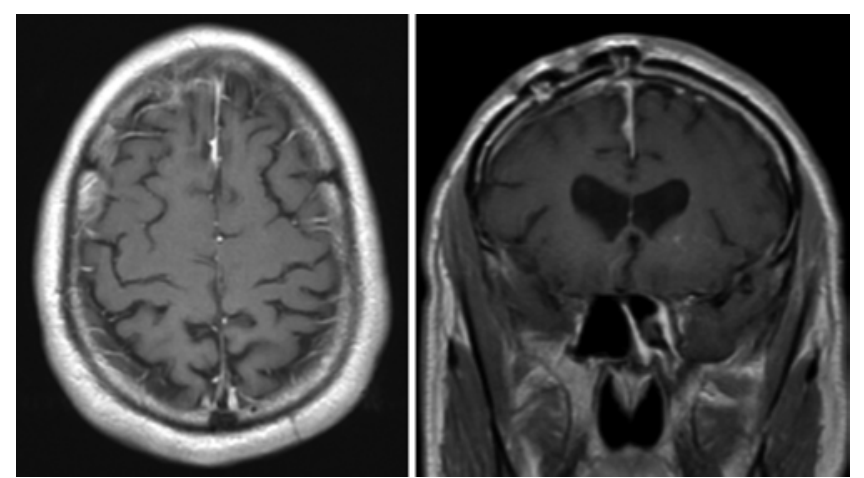

FIG. 8. Follow-up imaging 4 months after GKRS. Postcontrast axial (left) and coronal (right) MR images demonstrate stable enhancing disease at the posterior inferior tumor bed. 
in the absence of KMP. GKRS may be considered for recurrence.

\section{References}

1. Abe M, Misago N, Tanaka S, Masuoka J, Tabuchi K: Capillary hemangioma of the central nervous system: a comparative study with lobular capillary hemangioma of the skin. Acta Neuropathol 109:151-158, 2005

2. Chang JM, Kwon BJ, Han MH, Kang HS, Chang KH: Kaposiform hemangioendothelioma arising from the internal auditory canal. AJNR Am J Neuroradiol 27:931-933, 2006

3. Cho WS, Kim SK, Park SH, Cho BK: Intracranial kaposiform hemangioendothelioma: proposal of a new malignant variant. J Neurosurg Pediatr 3:147-150, 2009

4. Colmenero I, Hoeger PH: Vascular tumours in infants. Part II: vascular tumours of intermediate malignancy [corrected] and malignant tumours. Br J Dermatol 171:474-484, 2014

5. Croteau SE, Liang MG, Kozakewich HP, Alomari AI, Fishman SJ, Mulliken JB, et al: Kaposiform hemangioendothelioma: atypical features and risks of Kasabach-Merritt phenomenon in 107 referrals. J Pediatr 162:142-147, 2013

6. da Silva AD, de Oliveira Ramos G, Gomes RF, Martins MA, Lamers ML, Santa'Ana Filho M, et al: Tufted angioma in children: report of two cases and a review of the literature. Case Rep Dent 2014:942489, 2014

7. DeFatta RJ, Verret DJ, Adelson RT, Gomez A, Myers LL: Kaposiform hemangioendothelioma: case report and literature review. Laryngoscope 115:1789-1792, 2005

8. Enjolras O, Wassef M, Mazoyer E, Frieden IJ, Rieu PN, Drouet L, et al: Infants with Kasabach-Merritt syndrome do not have "true" hemangiomas. J Pediatr 130:631-640, 1997

9. Fernandez AP, Wolfson A, Ahn E, Maldonad JC, AlonsoLlamazares J: Kasabach-Merritt phenomenon in an adult man with a tufted angioma and cirrhosis responding to radiation, bevacizumab, and prednisone. Int J Dermatol 53:1165-1176, 2014

10. Hardisson D, Prim MP, De Diego JI, Patrón M, Escribano A, Rabanal I: Kaposiform hemangioendothelioma of the external auditory canal in an adult. Head Neck 24:614-617, 2002

11. Hunt SJ, Santa Cruz DJ: Vascular tumors of the skin: a selective review. Semin Diagn Pathol 21:166-218, 2004

12. Jones EW: Dowling Oration 1976. Malignant vascular tumours. Clin Exp Dermatol 1:287-312, 1976

13. Kelly M: Kasabach-Merritt phenomenon. Pediatr Clin North Am 57:1085-1089, 2010

14. Macmillan A, Champion RH: Progressive capillary haemangioma. Br J Derm 85:492-493, 1971
15. Mentzel T, Wollina U, Castelli E, Kutzner H: [Tufted hemangioma. Clinicopathologic and immunohistologic analysis of 5 cases of a distinct entity within the spectrum of capillary hemangioma.] Hautarzt 47:369-375, 1996 (Ger)

16. Mirza B, Shi WY, Phadke R, Holton JL, Turner C, Plant GT, et al: Strawberries on the brain-intracranial capillary hemangioma: two case reports and systematic literature review in children and adults. World Neurosurg 80:900.e13-900.e21, 2013

17. Nakagawa K: [Case report of angioblastoma of the skin.] Jpn J Dermatol 59:92-94, 1949 (Jpn)

18. North PE, Waner M, Mizeracki A, Mihm MC Jr: GLUT1: a newly discovered immunohistochemical marker for juvenile hemangiomas. Hum Pathol 31:11-22, 2000

19. O'Rafferty C, O'Regan GM, Irvine AD, Smith OP: Recent advances in the pathobiology and management of KasabachMerritt phenomenon. Br J Haematol 171:38-51, 2015

20. Pesapane F, Nazzaro G, Alberti-Violetti S, Gianotti R: A case of acquired tufted angioma in adulthood. An Bras Dermatol 90 (3 Suppl 1):16-18, 2015

21. Sarkar M, Mulliken JB, Kozakewich HP, Robertson RL, Burrows PE: Thrombocytopenic coagulopathy (Kasabach-Merritt phenomenon) is associated with Kaposiform hemangioendothelioma and not with common infantile hemangioma. Plast Reconstr Surg 100:1377-1386, 1997

\section{Disclosures}

Dr. Wang has served as a consultant to Abbvie, Merck, and Doximity.

\section{Author Contributions}

Conception and design: D'Amico, Zanazzi, Faust, McKhann. Acquisition of data: D'Amico, Zanazzi, Chan, McKhann. Analysis and interpretation of data: D'Amico, Zanazzi, Hargus, Dyster, Chan, Faust, McKhann. Drafting the article: D’Amico, Zanazzi, Faust. Critically revising the article: D’Amico, Zanazzi, LignelliDipple, Wang, Faust, McKhann. Reviewed submitted version of manuscript: D'Amico, Zanazzi, Faust. Approved the final version of the manuscript on behalf of all authors: D'Amico. Administrative/technical/material support: Wang, Faust, McKhann. Study supervision: Lignelli-Dipple, Wang, Faust, McKhann.

\section{Correspondence}

Randy S. D'Amico, Department of Neurological Surgery, Columbia University Medical Center, 710 W 168th St., New York, NY 10032.email: rsd9005@columbia.edu. 